\title{
Awake prone positioning for hypoxaemic respiratory failure: past, COVID-19 and perspectives
}

\author{
François Touchon ${ }^{1}$, Youssef Trigui ${ }^{1}$, Eloi Prud'homme ${ }^{2,3}$, Laurent Lefebvre ${ }^{4}$, Alais Giraud ${ }^{5}$, \\ Anne-Marie Dols ${ }^{6}$, Stéphanie Martinez ${ }^{1}$, Marie Bernardi ${ }^{1}$, Camille Begne ${ }^{1}$, Pascal Granier ${ }^{7}$, Pascal Chanez ${ }^{8}$, \\ Jean-Marie Forel ${ }^{2,3}$, Laurent Papazian ${ }^{2,3}$ and Xavier Elharrar ${ }^{1}$
}

\begin{abstract}
${ }^{1}$ Service des Maladies Respiratoires, CH Aix-en-Provence, Aix-en-Provence, France. ${ }^{2}$ Médecine Intensive Réanimation, Assistance Publique Hôpitaux de Marseille, Marseille, France. ${ }^{3}$ EA 3279, CEReSS - Centre d'Etudes et de Recherches sur les Services de Santé et qualité de vie, Aix-Marseille Université, Faculté de Médecine, Marseille, France. ${ }^{4}$ Service de Réanimation Polyvalente, $\mathrm{CH}$ Aix-enProvence, Aix-en-Provence, France. ${ }^{5}$ Unité de Recherche Clinique, $\mathrm{CH}$ Aix-en-Provence, Aix-en-Provence, France. ${ }^{6} \mathrm{CHU}$ Grenoble-Alpes, Grenoble, France. ${ }^{7}$ Médecine Physique et de Réadaptation, $\mathrm{CH}$ Aix-en-Provence, Aix-en-Provence, France. ${ }^{8} \mathrm{Clinique}$ des bronches, de l'allergie et du sommeil, AP-HM, Hôpital Nord, Marseille, France.
\end{abstract}

Corresponding author: François Touchon (frtouch@gmail.com)

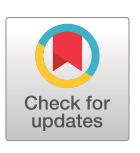

Copyright @The authors 2021

This version is distributed under the terms of the Creative Commons Attribution NonCommercial Licence 4.0. For commercial reproduction rights and permissions contact permissions@ersnet.org

Received: 27 Jan 2021 Accepted: 4 March 2021

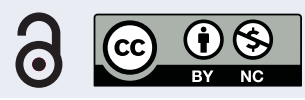

Shareable abstract (@ERSpublications)

In intubated ARDS patients, prone positioning reduces mortality. On spontaneously breathing patients, oxygenation improved during awake prone positioning. Relevant data on clinical outcomes are expected, especially on COVID-19 patients. https://bit.ly/2PU3B6V

Cite this article as: Touchon F, Trigui Y, Prud'homme E, et al. Awake prone positioning for hypoxaemic respiratory failure: past, COVID-19 and perspectives. Eur Respir Rev 2021; 30: 210022 [DOI: 10.1183/ 16000617.0022-2021].

\section{Abstract}

Prone positioning reduces mortality in the management of intubated patients with moderate-to-severe acute respiratory distress syndrome. It allows improvement in oxygenation by improving ventilation/perfusion ratio mismatching.

Because of its positive physiological effects, prone positioning has also been tested in non-intubated, spontaneously breathing patients, or "awake" prone positioning. This review provides an update on awake prone positioning for hypoxaemic respiratory failure, in both coronavirus disease 2019 (COVID-19) and non-COVID19 patients. In non-COVID-19 acute respiratory failure, studies are limited to a few small nonrandomised studies and involved patients with different diseases. However, results have been appealing with regard to oxygenation improvement, especially when combined with noninvasive ventilation or high-flow nasal cannula.

The recent COVID-19 pandemic has led to a major increase in hospitalisations for acute respiratory failure. Awake prone positioning has been used with the aim to prevent intensive care unit admission and mechanical ventilation. Prone positioning in conscious, non-intubated COVID-19 patients is used in emergency departments, medical wards and intensive care units.

Several trials reported an improvement in oxygenation and respiratory rate during prone positioning, but impacts on clinical outcomes, particularly on intubation rates and survival, remain unclear. Tolerance of prolonged prone positioning is an issue. Larger controlled, randomised studies are underway to provide results concerning clinical benefit and define optimised prone positioning regimens.

\section{Introduction}

Prone positioning consists of placing a patient face down. Prone positioning has been used for more than 40 years to improve oxygenation in patients who require invasive mechanical ventilation for acute respiratory distress syndrome (ARDS). The first demonstration of a beneficial effect on patients' oxygenation was shown in 1974 [1].

Numerous studies have demonstrated physiological improvement related to prone positioning. Nevertheless, these benefits have only recently been translated into improved patient outcomes. The first evidence of reduced mortality was reported by the PROSEVA study, in which early and prolonged prone positioning sessions (of at least $16 \mathrm{~h}$ ) were applied without increasing the rate of complications [2]. These 
results were later confirmed in several meta-analyses [3, 4]. In the management of moderate-to-severe ARDS, prone positioning is now strongly advocated [5].

Because of the positive physiological effects of prone positioning on transpulmonary pressure, lung compression and ventilation perfusion ratio, it has also been applied to non-intubated patients with acute respiratory failure (ARF), to improve oxygenation and delay or even avoid the need for invasive ventilation [6].

Data in spontaneously breathing patients were limited to retrospective studies or small prospective cohorts until the recent coronavirus disease 2019 (COVID-19) pandemic outbreak characterised by increased ARDS and intensive care unit (ICU) overload.

The aims of the present review were to summarise physiological aspects and rationale for using prone positioning in spontaneously breathing patients with hypoxaemic ARF, either with or without COVID-19.

\section{Physiological effects}

Available data on the physiological effects of prone positioning mainly concern mechanically ventilated patients.

Currently, described leading mechanisms are decreased lung compression in the gravity dependant zone, homogenisation of transpulmonary pressure, improvement of ventilation/perfusion ratio, and reduction of ventilator-induced lung injury (VILI) or patient self-inflicted lung injury (P-SILI) (figure 1).

\section{Decreased lung compression}

In the supine position, the dorsal part of the lung is compressed by its own weight, the mediastinum weight, the abdominal compartment and the diaphragm weight.

The effects of prone and supine positioning on lung density have been compared by GATTINONi et al. [7], using computerised tomography, in patients with ARDS. This study found that prone positioning redistributed lung densities from the dorsal to ventral regions. This finding led to the "sponge lung model", where ARDS was characterised by a heavy oedematous lung that can be compared to a wet sponge.

By placing the patient in the prone position, the lung's compression due to its own weight is reduced via a gravitational-dependent redistribution of fluids. In addition, the weight of the mediastinum is supported by the sternum, the stiffer part of the chest. At the same time, the diaphragm is displaced caudally, decreasing compression of the posterior-caudal lung parenchyma [8]. Finally, in a triangular-shaped lung, more parenchyma is included in the dorsal half than in the ventral one resulting in a more aerated lung in prone positioning [9].

\section{Homogenisation of transpulmonary pressure}

During spontaneous breathing, the inspiratory effort exerted by respiratory muscles decreases pleural pressure further, giving rise to pulmonary ventilation. This pressure gradient across the lung is named transpulmonary pressure $\left(P_{\mathrm{L}}\right)$, i.e. the pressure distending the lung. $P_{\mathrm{L}}$ is defined as the difference between the airway/alveolar pressure and pleural pressure estimated by oesophageal pressure: $P_{\mathrm{L}}=$ airway/alveolar pressure - pleural pressure.
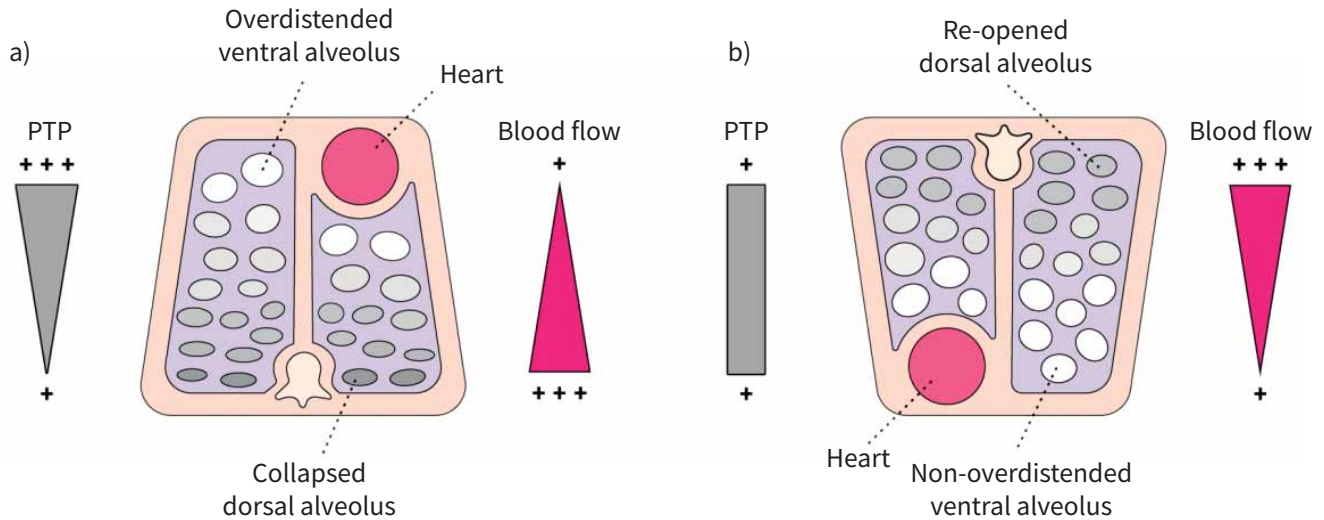

FIGURE 1 Effects of a) supine positioning and b) prone positioning on lung mechanics. 
In mechanical ventilation, variations in $P_{\mathrm{L}}$ (and variations in lung compliance) are used to define variations in lung volume. For example, for a same level of lung compliance, the larger the $P_{\mathrm{L}}$, the larger the lung volume.

With the patient in the supine position, the dorsal pleural pressure is greater than ventral pleural pressure. As a result, the ventral $P_{\mathrm{L}}$ exceeds the dorsal $P_{\mathrm{L}}$. The higher $P_{\mathrm{L}}$ at the ventral part of the lung leads to hyperinflation, while the lower $P_{\mathrm{L}}$ at the dorsal part of the lung leads to atelectasis. Prone positioning reduces the difference between the dorsal and ventral $P_{\mathrm{L}}$, leading to a decrease in ventral alveolar hyperinflation and dorsal alveolar collapse [10]. These mechanisms are increased in patients with ARDS, where the dorsal part of the lung is heavier (due to inflammation, oedema in the sloping areas and weight of the overlying lung), and the tidal volume is preferably distributed to the ventral, nondependent part of the lungs [6].

\section{Ventilation/perfusion ratio improvement}

Ever since the work of WEST et al. [11] in 1964, we have known that, in healthy volunteers, pulmonary perfusion is greater in the lung bases in the upright position and in the dorsal zones of the lung when in the supine position.

Blood flow is independent of the gravitational gradient. In the prone position, the dorsal part continues to receive the majority of the blood flow when alveoli reopen, while the ventral part continues to receive the minority of the blood flow when alveoli collapse [12]. There is, therefore, a decrease in the related intra-pulmonary shunt and an improvement in the ventilation/perfusion ratio [7].

In patients with ARDS, the gas exchange impairment is mainly related to disturbed ventilation/perfusion ratio [13], the management of hypoxaemia is clearly devoted to its improvement.

\section{Reduction of VILI and P-SILI}

In mechanically ventilated ARDS patients, prone positioning can also prevent VILI, an acute lung injury caused or worsened by mechanical ventilation. VILI includes several lung mechanic alterations that can lead to worsening hypoxaemia and increased mortality, especially in ARDS patients. Adopting a protective mechanical ventilation strategy can prevent VILI and improve survival [14]. This strategy includes an increase in positive end-expiratory pressure (PEEP) to prevent alveolar de-recruitment but may also favour overdistension of previously well-ventilated alveoli [15]. Adding prone positioning to high PEEP may decrease regional overdistension and small airways opening/closing events, thereby preventing VILI [16].

Similarly to intubated ARDS patients, spontaneously breathing patients with hypoxaemic ARF can generate high respiratory drives and forceful inhalation effort, leading to lung damage similar to VILI [17]. This concept is termed P-SILI and worsens respiratory mechanics and haematosis, leading to an increased respiratory drive and increased P-SILI, as a vicious cycle [18]. Decreasing inspiratory effort by improving gas exchange in the prone position could be an option to reduce P-SILI during ARF.

\section{Prone positioning in conscious, non-intubated, non-COVID-19 patients}

Evidence of prone positioning benefits in non-intubated patients is limited to a few case reports and small observational studies (table 1).

\section{Effects on oxygenation}

The first use of prone positioning in spontaneously breathing patients was described in 1999, in two studies including paediatric patients, in whom oxygenation was improved during prone positioning [19, 20]. In four adults with hypoxaemic respiratory failure, arterial oxygen tension $\left(\mathrm{PaO}_{2}\right)$ improved after the first session of prone positioning [21]. Two other studies tested awake prone positioning in patients with severe hypoxaemia after lung transplantation, in addition to noninvasive ventilation (NIV) or high-frequency percussive ventilation. Improvement of oxygenation and respiratory mechanics have been reported after multiple sessions [22, 23].

\section{Prone positioning in addition to respiratory devices}

A first retrospective observational study based on a 5-year experience included 15 ARF patients managed with high-flow nasal cannula (HFNC) or NIV, performing multiple prone positioning sessions. $P_{\mathrm{aO}_{2}}$ improved significantly during pronation [24]. A second prospective observational study [25] recruited 20 patients receiving, similarly, awake prone positioning in addition to HFNC and/or NIV for moderate-to-severe, infectious, ARDS. $P_{\mathrm{aO}_{2}}$ inspiratory oxygen fraction $\left(F_{\mathrm{IO}_{2}}\right)$ improved and intubation 
TABLE 1 Prone positioning in non-intubated, non-coronavirus disease 2019 patients

\begin{tabular}{|c|c|c|c|c|c|c|c|}
\hline First author [ref.] & $\begin{array}{c}\text { Patients } \\
\mathrm{n}\end{array}$ & Diseases & $\begin{array}{l}\text { Respiratory } \\
\text { device }\end{array}$ & $\begin{array}{l}\mathrm{PP} \\
\text { regimen }\end{array}$ & Main results & $\begin{array}{l}\text { Adverse } \\
\text { event }\end{array}$ & Comments \\
\hline $\begin{array}{l}\text { CHAISUPAMONGKOLLARP } \\
\text { [19] }\end{array}$ & 17 & Pneumonia & Oxygen & & $\begin{array}{c}\text { Improve } S_{\mathrm{pO}_{2}} \\
\text { Improve compliance }\end{array}$ & & Paediatric \\
\hline TuLLeKEN $[20]$ & 1 & Pneumonia & HFNC & $20 \mathrm{~h}$ & $\begin{array}{l}\text { Improve } P_{\mathrm{aO}} \\
\text { Resolve opacities }\end{array}$ & 0 & Paediatric \\
\hline Valter [21] & 4 & $\begin{array}{c}\text { CHF, pneumonia, } \\
\text { ARDS }\end{array}$ & 0 & $1-5 \mathrm{~h}$ & $\begin{array}{c}\text { Improve } P_{\mathrm{aO}} \\
\text { Avoid intubation }\end{array}$ & 0 & $\begin{array}{l}1 \text { death } \\
\text { (CHF) }\end{array}$ \\
\hline Feltracco [22] & 2 & $\begin{array}{l}\text { Post-reimplantation } \\
\text { syndrome }\end{array}$ & NIV & $\begin{array}{c}6-8 \mathrm{~h} \\
\text { per day, } \\
8-10 \text { days }\end{array}$ & $\begin{array}{l}\text { Decrease } F_{\mathrm{IO}_{2}} \\
\text { NIV removal }\end{array}$ & 0 & \\
\hline Feltracco [23] & 3 & $\begin{array}{l}\text { Lung transplant } \\
\text { respiratory } \\
\text { complications }\end{array}$ & HFPV & $\begin{array}{c}1-3 \mathrm{~h}, \\
3-6 \text { times } \\
\text { per day }\end{array}$ & $\begin{array}{c}\text { Improve secretions } \\
\text { clearance } \\
\text { Improve respiratory } \\
\text { mechanic } \\
\text { Attenuate graft infiltrates } \\
\text { Improve gas exchange }\end{array}$ & 0 & \\
\hline SCARAVILLI [24] & 15 & $\begin{array}{c}\text { Hypoxaemic acute } \\
\text { respiratory failure } \\
\text { (pneumonia, fascitis, } \\
\text { sepsis) }\end{array}$ & $\begin{array}{l}\text { HFNC, CPAP } \\
\text { and NIV }\end{array}$ & $\begin{array}{c}3 \mathrm{~h} \\
\text { per day, } \\
2 \text { days }\end{array}$ & Improve $P_{\mathrm{aO}}$ & $\begin{array}{c}\text { Intolerance } \\
\qquad(n=2)\end{array}$ & $\begin{array}{l}\text { Retrospective } \\
\mathrm{PaO}_{2} \text { return to } \\
\text { baseline after } \\
\text { resupination }\end{array}$ \\
\hline DING [25] & 20 & $\begin{array}{c}\text { Infectious, } \\
\text { moderate-to-severe } \\
\text { ARDS (pneumonia, } \\
\text { influenza) }\end{array}$ & $\begin{array}{l}\text { NIV and } \\
\text { HFNC }\end{array}$ & $\begin{array}{l}30 \text { min } \\
\text { twice } \\
\text { per day, } \\
\geqslant 3 \text { days }\end{array}$ & $\begin{array}{l}\text { Avoid intubation } \\
\text { Improve } P_{\mathrm{aO}_{2}} / F_{\mathrm{IO}_{2}}\end{array}$ & $\begin{array}{l}\text { Intolerance } \\
\qquad(n=2)\end{array}$ & $\begin{array}{l}\text { Intubation in } \\
78 \% \text { of severe } \\
\text { ARDS patients }\end{array}$ \\
\hline Pérez-Nieto [26] & 6 & $\begin{array}{c}\text { Non-infectious, } \\
\text { severe ARDS } \\
\text { (thoracic trauma, } \\
\text { lupus pneumonitis) }\end{array}$ & $\begin{array}{l}\text { NIV and } \\
\text { HFNC }\end{array}$ & $\begin{array}{l}2-3 \mathrm{~h} \\
\text { per } 12 \mathrm{~h}, \\
2 \text { days }\end{array}$ & Avoid intubation & 0 & Retrospective \\
\hline
\end{tabular}

PP: prone positioning; $\mathrm{S}_{\mathrm{pO}_{2}}$ : oxygen saturation measured by pulse oximetry; HFNC: high-flow nasal cannula; $P_{\mathrm{aO}}$ : arterial oxygen tension; $\mathrm{CHF}$ : congestive heart failure; ARDS: acute respiratory distress syndrome; NIV: noninvasive ventilation; $F_{\mathrm{IO}_{2}}$ : inspiratory oxygen fraction; HFPV: high-frequency percussive ventilation; CPAP: continuous positive airway pressure.

could be avoided in up to half of the patients. Most severe patients finally required intubation suggesting they were not appropriate candidates for prone positioning trials.

Together, these studies suggest positive effects of prone positioning in awake, non-intubated patients on oxygen, especially when combined with NIV or HFNC. However, it remains unclear if these benefits persist after resupination and if they lead to improved clinical outcomes.

\section{Prone positioning in conscious, non-intubated COVID-19 patients}

The COVID-19 pandemic led to a dramatic increase in the number of patients requiring respiratory support for ARDS, resulting in an ICU overload. Therefore, clinicians have to use innovative approaches to limit the need for mechanical ventilation, including awake prone positioning.

The first use of prone positioning in the management of non-intubated COVID patients was briefly described at the beginning of the pandemic in China [27], where prone positioning was part of a bundled intervention, including NIV, HFNC and restrictive fluid resuscitation. Authors described a decreased need for invasive mechanical ventilation, with an intubation rate $<1 \%$ among their hospitalised patients.

Given these promising results, numerous studies were performed around the world to assess the impact of prone positioning on oxygenation when combined with conventional oxygen therapy (COT), HFNC or NIV. Other authors studied the possible role of prone positioning as a prognostic factor and the impact on clinical outcomes, particularly intubation rate (table 2).

\section{Effects on oxygenation}

ELHARRAR et al. [28] conducted a prospective, single-centre study of prone positioning in patients with hypoxaemic ARF requiring oxygen supplementation with HFNC or COT. Among the 24 patients included, 
TABLE 2 Prone positioning in non-intubated, coronavirus disease 2019 (COVID-19) patients

\begin{tabular}{|c|c|c|c|c|c|c|c|}
\hline $\begin{array}{l}\text { First author } \\
\text { [ref.] }\end{array}$ & Design & $\begin{array}{c}\text { Patients } \\
\mathrm{n}\end{array}$ & $\begin{array}{l}\text { Respiratory } \\
\text { device }\end{array}$ & PP regimen & Main results & Adverse event & Comments \\
\hline Sun [27] & $\begin{array}{c}\text { Retrospective } \\
\text { analysis }\end{array}$ & 631 & $\begin{array}{l}\text { HFNC and } \\
\text { NIV }\end{array}$ & Unknown & $\begin{array}{c}\text { Combined } \\
\text { strategy } \\
\text { including PP } \\
\text { might decrease } \\
\text { mortality }\end{array}$ & & $\begin{array}{l}\text { Bundled intervention } \\
\text { PP regimen undisclosed }\end{array}$ \\
\hline $\begin{array}{c}\text { ELHARRAR } \\
{[28]}\end{array}$ & $\begin{array}{c}\text { Prospective } \\
\text { feasibility study }\end{array}$ & 24 & $\begin{array}{l}\text { COT and } \\
\text { HFNC }\end{array}$ & $\begin{array}{l}\geqslant 3 \mathrm{~h} \text {, one } \\
\text { session }\end{array}$ & $\begin{array}{l}\text { Increased } P_{\mathrm{aO}_{2}} \\
\text { during PP }\end{array}$ & Back pain $(42 \%)$ & $\begin{array}{l}3 \text { patients maintained } \\
\text { improvement after } \\
\text { resupination }\end{array}$ \\
\hline Despres [29] & $\begin{array}{c}\text { Prospective } \\
\text { feasibility study }\end{array}$ & 6 & $\begin{array}{l}\text { COT and } \\
\text { HFNC }\end{array}$ & 1 to $16 \mathrm{~h}$ & Improve $P_{\mathrm{aO}_{2}} / F_{\mathrm{IO}_{2}}$ & & $\begin{array}{l}\text { In ICU } 3(50 \%) \text { patients } \\
\text { required intubation }\end{array}$ \\
\hline $\mathrm{Xu}[30]$ & $\begin{array}{l}\text { Retrospective } \\
\text { case series }\end{array}$ & 10 & HFNC & 16 h per day & Improve $P_{\mathrm{aO}_{2}} / \mathrm{F}_{\mathrm{IO}_{2}}$ & $\begin{array}{l}\text { Discomfort and } \\
\text { anxiety }\end{array}$ & $\begin{array}{c}0 \text { patients required } \\
\text { intubation }\end{array}$ \\
\hline $\mathrm{NG}[31]$ & $\begin{array}{l}\text { Prospective } \\
\text { case series }\end{array}$ & 10 & COT & $\begin{array}{l}1 \mathrm{~h}, 5 \text { times } \\
\text { per day }\end{array}$ & $\begin{array}{l}\text { Shortening of } \\
\text { oxygen weaning }\end{array}$ & $\begin{array}{l}\text { Musculoskelatal } \\
\text { discomfort and } \\
\text { nausea/vomiting }\end{array}$ & $\begin{array}{c}8 \text { patients received } \\
\text { COVID-19 specific } \\
\text { therapies }\end{array}$ \\
\hline DamarLa [32] & $\begin{array}{l}\text { Retrospective } \\
\text { case series }\end{array}$ & 10 & $\begin{array}{l}\text { COT and } \\
\text { HFNC }\end{array}$ & $\begin{array}{l}\text { Alternate every } \\
\qquad 2 \mathrm{~h}\end{array}$ & $\begin{array}{c}\text { Improve } S_{\mathrm{pO}_{2}} \\
\text { Decrease } \\
\text { dyspnoea/RR }\end{array}$ & & $\begin{array}{l}\text { In ICU } 2 \text { patients } \\
\text { required intubation } \\
\text { (most severe, } \\
\text { HFNC } \\
0.5 \text { and } 0.6 \text { ) }\end{array}$ \\
\hline Reddy [33] & Meta-analysis & 449 & $\begin{array}{l}\text { COT, HFNC } \\
\text { and NIV }\end{array}$ & Heterogeneous & $\begin{array}{c}\text { Improve } \\
\text { oxygenation } \\
\text { Decrease RR }\end{array}$ & & Included 15 studies \\
\hline SARTINI [34] & $\begin{array}{l}\text { Prospective } \\
\text { case series }\end{array}$ & 15 & CPAP & $\begin{array}{l}\text { Based on } \\
\text { severity and } \\
\text { adherence }\end{array}$ & $\begin{array}{c}\text { Improve } S_{\mathrm{pO}_{2}} \text { and } \\
P_{\mathrm{aO}_{2}} / F_{\mathrm{IO}_{2}} \\
\text { Decrease RR }\end{array}$ & & $\begin{array}{l}80 \% \text { of patients } \\
\text { maintain oxygenation } \\
\text { improvement } \\
\text { after } \\
\text { resupination }\end{array}$ \\
\hline Coppo [35] & $\begin{array}{c}\text { Prospective } \\
\text { feasibility study }\end{array}$ & 56 & $\begin{array}{l}\text { Helmet } \\
\text { CPAP }\end{array}$ & $\geqslant 3 \mathrm{~h}$ & Improve $P_{\mathrm{aO}_{2}} / F_{\mathrm{IO}_{2}}$ & $\begin{array}{l}\text { Discomfort and } \\
\text { cough }\end{array}$ & $\begin{array}{l}50 \% \text { of patients } \\
\text { maintain } P_{\mathrm{aO}_{2}} / F_{\mathrm{IO}_{2}} \\
\text { improvement after } \\
\text { resupination but no } \\
\text { difference on } \\
\text { intubation rate }\end{array}$ \\
\hline CAPUTO [36] & $\begin{array}{l}\text { Observational } \\
\text { cohort study }\end{array}$ & 50 & COT & $5 \mathrm{~min}$ & Improve $S_{\mathrm{pO}_{2}}$ & & $\begin{array}{c}\text { In ED } 13(25 \%) \\
\text { patients required } \\
\text { intubation } \\
\text { within } 24 \mathrm{~h}\end{array}$ \\
\hline $\begin{array}{c}\text { Thompson } \\
\text { [37] }\end{array}$ & $\begin{array}{c}\text { Prospective } \\
\text { feasibility study }\end{array}$ & 25 & COT & $1 \mathrm{~h}$ & Improve $S_{\mathrm{pO}_{2}}$ & & $\begin{array}{c}\text { In IMCU } 12(48 \%) \\
\text { patients required } \\
\text { intubation } \\
\text { Patients with an } \mathrm{S}_{\mathrm{pO}_{2}} \\
\geqslant 95 \% \text { after } 1 \mathrm{~h} \text { of } \mathrm{PP} \\
\text { was associated with a } \\
\text { lower rate of intubation }\end{array}$ \\
\hline Hallifax [38] & $\begin{array}{l}\text { Retrospective } \\
\text { cohort study }\end{array}$ & 30 & $\begin{array}{l}\text { HFNC and } \\
\text { NIV }\end{array}$ & $\begin{array}{c}2 \mathrm{~h} \text { twice } \\
\text { per day, } \\
2 \text { consecutive } \\
\text { days }\end{array}$ & $\begin{array}{l}\text { Reduced } \\
\text { mortality }\end{array}$ & & $\begin{array}{l}\text { In ICU only } 11 \text { patients } \\
\text { "fully" prone }\end{array}$ \\
\hline PAdRÃo [39] & $\begin{array}{l}\text { Retrospective } \\
\text { cohort study }\end{array}$ & 57 & COT & $\geqslant 4 \mathrm{~h}$ & $\begin{array}{l}\text { Do not reduce } \\
\text { intubation rate }\end{array}$ & $\begin{array}{l}\text { Accidental removal } \\
\text { of i.v. lines }\end{array}$ & $\begin{array}{c}\text { Only COT was used } \\
\text { while other respiratory } \\
\text { devices might improve } \\
\text { outcomes }\end{array}$ \\
\hline $\begin{array}{c}\text { FERRANDO } \\
{[40]}\end{array}$ & $\begin{array}{l}\text { Prospective } \\
\text { cohort study }\end{array}$ & 55 & HFNC & $16 \mathrm{~h}$ per day & $\begin{array}{l}\text { Do not reduce } \\
\text { intubation rate }\end{array}$ & & $\begin{array}{l}\text { In ICU did not establish } \\
\text { whether was used as a } \\
\text { routine or life-saving } \\
\text { therapy } \\
\text { Non-prone group also } \\
\text { included regimen }<16 \mathrm{~h} \\
\text { per day }\end{array}$ \\
\hline
\end{tabular}




\begin{tabular}{|c|c|c|c|c|c|c|c|}
\hline $\begin{array}{l}\text { First author } \\
\text { [ref.] }\end{array}$ & Design & $\begin{array}{c}\text { Patients } \\
\mathrm{n}\end{array}$ & $\begin{array}{l}\text { Respiratory } \\
\text { device }\end{array}$ & PP regimen & Main results & Adverse event & Comments \\
\hline JaGAN [41] & $\begin{array}{l}\text { Retrospective } \\
\text { analysis }\end{array}$ & 40 & & $\begin{array}{c}\geqslant 1 \mathrm{~h}, 5 \text { times per } \\
\quad \text { day, }+1 \mathrm{~h} \\
\text { overnight }\end{array}$ & $\begin{array}{c}\text { Decrease } \\
\text { intubation rate }\end{array}$ & & $\begin{array}{c}\text { Respiratory devices } \\
\text { undisclosed } \\
\text { Prone group patient } \\
\text { were younger and } \\
\text { healthier } \\
\text { PP regimen at patient's } \\
\text { discretion }\end{array}$ \\
\hline
\end{tabular}

PP: prone positioning; HFNC: high-flow nasal cannula; NIV: noninvasive ventilation; COT: conventional oxygen therapy; $P_{\mathrm{aO}}$ : arterial oxygen tension; $F_{\mathrm{IO}_{2}}$ : inspiratory oxygen fraction; ICU: intensive care unit; $\mathrm{S}_{\mathrm{DO}_{2}}$ : oxygen saturation measured by pulse oximetry; RR: respiratory rate; CPAP: continuous positive airway pressure; ED: emergency department; IMCU: intermediate care unit.

15 (63\%) were able to tolerate a first prone positioning session for at least $3 \mathrm{~h}$. Among the tolerant patients, $P_{\mathrm{aO}_{2}}$ increased from $73.6 \mathrm{mmHg}$ before to $94.9 \mathrm{mmHg}$ during prone positioning. Only three patients maintained improved oxygenation 6-12 h after resupination. In COVID-19 hospitalised patients other similar studies have reported improved oxygenation during prone positioning, with a heterogenous prone positioning regimen [29-32].

A total of 15 studies, representing 449 patients, have been included in a systematic review and meta-analysis [33], assessing the change in oxygenation (i.e. $P_{\mathrm{aO}_{2}} / F_{\mathrm{IO}_{2}}$ ratio, $P_{\mathrm{aO}_{2}}$ and oxygen saturation measure by pulse oximetry $\left(S_{\mathrm{pO}_{2}}\right)$ ) after prone positioning. Despite heterogeneity in prone positioning regimens and associated respiratory devices, significant oxygenation improvement was reported at the end of the prone positioning session.

\section{Prone positioning in addition to respiratory devices}

Prone positioning was also used in addition to NIV/continuous positive airway pressure (CPAP), in two Italian studies. In the first [34], 15 patients who were hypoxaemic despite a $10 \mathrm{cmH}_{2} \mathrm{O}$ CPAP were placed

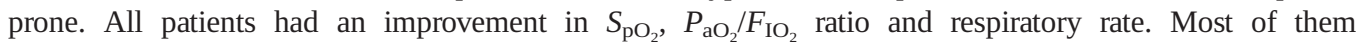
maintained the oxygenation improvement after prone positioning. In addition to helmet CPAP, prone positioning was used for a minimum duration of $3 \mathrm{~h}$. The $P_{\mathrm{aO}_{2}} / F_{\mathrm{IO}_{2}}$ ratio significantly improved, and improvement was sustained in half of the patients after resupination [35].

\section{Tolerance of prone positioning as a prognostic factor}

Few trials reported a possible role of prone position as a prognostic factor for COVID-19 patients. A first retrospective study [36], was conducted in a New York City (NY, USA) emergency department and involved 50 patients with hypoxia at triage. The authors reported a significant increase in $S_{\mathrm{pO}_{2}}$ during prone positioning [36]. Failure to improve oxygenation during initial proning seemed to be associated with an increased risk of intubation. In the second study [37], 25 hypoxaemic patients in an intermediate care unit presented with a significant $S_{\mathrm{pO}_{2}}$ increase during prone positioning. A lower rate of intubation was observed in patients with $S_{\mathrm{pO}_{2}} \geqslant 95 \%$ after $1 \mathrm{~h}$ of proning. More recently, a retrospective study [38] included 48 patients requiring CPAP and/or HFNC. Only 11 patients could tolerate prone positioning for at least 2 hours twice daily for two consecutive days (full proning), but achievement of full proning was associated with reduced mortality.

\section{Effects on clinical outcomes}

Several studies have specifically assessed whether the use of awake prone positioning is associated with improved clinical outcomes, and particularly reduced intubation rate. A single-centre retrospective study [39] compared awake prone positioning to usual care in 57 hypoxaemic patients presenting with increased work of breathing (i.e. tachypnoea $\geqslant 24$ breaths $\cdot \mathrm{min}^{-1}$ ). Exposure to awake prone positioning was not associated with reduced intubation rate. Then, in a multicentre prospective study [40], prone positioning was used as adjunctive therapy to HFNC in 55 patients compared to 144 patients. Intubation rate was not lower in the prone group but prone positioning was only considered when applied for $\geqslant 16 \mathrm{~h} \cdot \mathrm{day}^{-1}$. Awake prone positioning for $<16 \mathrm{~h} \cdot$ day $^{-1}$ could have reduced the risk of intubation in the control group. Finally, a retrospective analysis [41] compared 40 patients who could be proned to those who could not (65 patients). Intubation rate was significantly lower in the prone group, with a lower risk of intubation of $69 \%$. The effect of awake prone positioning on nosocomial pneumonia prevalence remains unknown. 
In an exposed/non-exposed bicentric retrospective matched cohort study, PRUD'HOMmE et al. [42] compared a prone group and a control group (no prone positioning). 96 COVID-19 patients with acute hypoxaemic respiratory failure requiring oxygen supplementation were included. Prone positioning for at least $3 \mathrm{~h}$ a day during three consecutive days may be associated with a clinical benefit by preventing the upgrading of oxygen delivery method.

Several conclusions can be drawn cautiously from these first trials in COVID-19: prone positioning improves oxygenation during proning. Scientific evidence on the prognostic value of initial awake prone positioning and on clinical outcomes are limited and require future trials.

\section{Limitations}

Strong clinical evidence for prone positioning is restricted to patients undergoing invasive mechanical ventilation. The application of prone positioning in awake, spontaneously breathing patients has not been largely studied. Several limitations can be identified.

Prone positioning in conscious patients might be a complementary tool to improve oxygenation in addition to respiratory devices as COT, HFNC, CPAP or NIV. Numerous studies have shown that prone positioning improved oxygenation, at least during prone positioning.

However, available data from trials using different methodologies and designs are contradictory on clinical outcomes. Lack of evidence on clinical benefits could be explained by the absence of large randomised controlled trials and because awake prone positioning sessions were much shorter than the prone positioning sessions recommended in intubated ARDS patients.

Most of the complications described during prone positioning in mechanically ventilated patients (airway obstruction, unplanned extubation, pressure ulcers, facial oedema, and brachial plexus neuropathy) seem to be related to sedation and mechanical ventilation. Other complications such as transient oxygen desaturation and loss of venous access are described in awake proning. Data are limited but no severe adverse events were described in published awake prone positioning studies.

Data are limited but no severe adverse events were described in published awake prone positioning studies. Nevertheless, some trials reported a limited tolerance of awake prone positioning sessions, with frequent side-effects: musculoskeletal discomfort [28, 30, 31, 35], nausea/vomiting [31], cough [35] or anxiety [30]. More than the duration of a single prone positioning course, multiple short prone positioning sessions in the presence of trained and experienced staff might be a solution to improve tolerance in awake patients and lead to clinical benefits. Published data do not allow us to determine for which patients prone positioning may be beneficial, or the best duration and frequency of prone positioning sessions [43].

\section{Clinical practical issues}

Before the first prone positioning session, explain the procedure to the patient. Ensure respiratory devices and intravenous lines are secure. For the first session, initial close monitoring $\left(S_{\mathrm{pO}_{2}}\right.$ and respiratory rate) should be performed. A call bell should be available. Frequent repositioning might upgrade tolerance. Pillows can be used to improve comfort. A nurse or a physical therapist may help positioning.

\section{Conclusion}

Awake prone positioning improves oxygenation during prone positioning, but persistence after resupination and impact on clinical outcomes remain undetermined.

Due to its relative ease of use, and low sides-effects, prone positioning for non-intubated patients has been widely applied and studied in COVID-19 patients, whether in medical wards or in emergency rooms. Association with NIV or HFNC is suggested to improve the benefits on respiratory status.

Findings on oxygenation are encouraging, but evidence is lacking on clinical outcome, such as mortality or intubation rates.

Studies do not rule on the best duration and frequency of prone positioning session, and tolerance of prolonged prone positioning sessions is a concern.

Many questions remain unanswered, and a systemic approach is needed. Numerous randomised trials, including a meta-trial [44], are in progress to assess the clinical benefits of prone positioning in the management of COVID patients (www.clinicaltrials.gov/ NCT04325906, NCT04347941, NCT04358939, 
NCT04395144). In the near future, awake prone positioning could be a simple, low-cost tool for the management of hypoxaemic ARF in spontaneously breathing, non-COVID and COVID patients.

Provenance: Submitted article, peer reviewed

Conflict of interest: F. Touchon has nothing to disclose. Y. Trigui has nothing to disclose. E. Prud'homme has nothing to disclose. L. Lefebvre has nothing to disclose. A. Giraud has nothing to disclose. A-M. Dols has nothing to disclose. S. Martinez has nothing to disclose. M. Bernardi has nothing to disclose. C. Begne has nothing to disclose. P. Granier has nothing to disclose. P. Chanez reports grants and personal fees from Almirall, Boehringer Ingelheim, ALK, GSK, AstraZeneca, Novartis, Teva and Chiesi, and grants from AMU, outside the submitted work. J-M. Forel has nothing to disclose. L. Papazian has nothing to disclose. X. Elharrar has nothing to disclose.

\section{References}

$1 \quad$ Bryan A. Conference on the scientific basis of respiratory therapy. Pulmonary physiotherapy in the pediatric age group. Comments of a devil's advocate. Am Rev Respir Dis 1974; 110: 143-144.

2 Guérin C, Reignier J, Richard JC, et al. Prone positioning in severe acute respiratory distress syndrome. N Eng J Med 2013; 368: 2159-2168.

3 Munshi L, Del Sorbo L, Adhikari NKJ, et al. Prone position for acute respiratory distress syndrome: a systematic review and meta-analysis. Ann Am Thorac Soc 2017; 14: Suppl. 4, S280-S288.

4 Park SY, Kim HJ, Yoo KH, et al. The efficacy and safety of prone positioning in adults patients with acute respiratory distress syndrome: a meta-analysis of randomised controlled trials. J Thorac Dis 2015; 7: 356-367.

5 Fan E, Del Sorbo L, Goligher EC, et al. An Official American Thoracic Society/European Society of Intensive Care Medicine/Society of Critical Care Medicine Clinical Practice Guideline: mechanical ventilation in adult patients with acute respiratory distress syndrome. Am J Respir Crit Care Med 2017; 195: 1253-1263.

6 Pelosi P, Brazzi L, Gattinoni L. Prone position in acute respiratory distress syndrome. Eur Respir J 2002; 20: 1017-1028.

7 Gattinoni L, Pelosi P, Vitale G, et al. Body position changes redistribute lung computed-tomographic density in patients with acute respiratory failure. Anesthesiology 1991; 74: 15-23.

8 Agostoni E, Mead J. Statics of the respiratory system. In: Fenn WO, Rahn H, Eds. Handbook of Physiology Respiration. Vol. 1. Washington DC, 1964, American Physiological Society, 387-409.

9 Gattinoni L, Pesenti A, Carlesso E. Body position changes redistribute lung computed-tomographic density in patients with acute respiratory failure: impact and clinical fallout through the following 20 years. Intensive Care Med 2013; 39: 1909-1915.

10 Kumaresan A, Gerber R, Mueller A, et al. Effects of prone positioning on transpulmonary pressures and end-expiratory volumes in patients without lung disease. Anesthesiology 2018; 128: 1187-1192.

11 West JB, Dollery CT, Naimark A. Distribution of blood flow in isolated lung; relation to vascular and alveolar pressures. J Appl Physiol 1964; 19: 713-724.

12 Nyrén $\mathrm{S}$, Mure $\mathrm{M}$, Jacobsson $\mathrm{H}$, et al. Pulmonary perfusion is more uniform in the prone than in the supine position: scintigraphy in healthy humans. J Appl Physiol 1999; 86: 1135-1141.

13 Radermacher P, Maggiore SM, Mercat A. Fifty years of research in ARDS. Gas exchange in acute respiratory distress syndrome. Am J Respir Crit Care Med 2017; 196: 964-984.

14 Acute Respiratory Distress Syndrome Network, Brower RG, Matthay MA, et al. Ventilation with lower tidal volumes as compared with traditional tidal volumes for acute lung injury and the acute respiratory distress syndrome. N Eng J Med 2000; 342: 1301-1308.

15 Gattinoni L, Mascheroni D, Torresin A, et al. Morphological response to positive end expiratory pressure in acute respiratory failure. Computerised tomography study. Intensive Care Med 1986; 12: 137-142.

16 Cornejo RA, Díaz JC, Tobar EA, et al. Effects of prone positioning on lung protection in patients with acute respiratory distress syndrome. Am J Respir Crit Care Med 2013; 188: 440-448.

17 Brochard L, Slutsky A, Pesenti A. Mechanical ventilation to minimize progression of lung injury in acute respiratory failure. Am J Respir Crit Care Med 2016; 195: 438-442.

18 Carteaux G, Perier F, Maraffi T, et al. Patient self-inflicted lung injury: what the intensivist needs to know. Méd Intensive Réa 2019; 28: 11-20.

19 Chaisupamongkollarp T, Preuthipan A, Vaicheeta S, et al. Prone position in spontaneously breathing infants with pneumonia. Acta Paediatr 1999; 88: 1033-1034.

20 Tulleken JE, van der Werf TS, Ligtenberg JJM, et al. Prone position in a spontaneously breathing near-drowning patient. Intensive Care Med 1999; 25: 1469-1470.

21 Valter C, Christensen AM, Tollund C, et al. Response to the prone position in spontaneously breathing patients with hypoxemic respiratory failure. Acta Anaesthesiol Scand 2003; 47: 416-418.

22 Feltracco P, Serra E, Barbieri S, et al. Non-invasive ventilation in prone position for refractory hypoxemia after bilateral lung transplantation. Clin Transpl 2009; 23: 748-750. 
23 Feltracco P, Serra E, Barbieri S, et al. Noninvasive high-frequency percussive ventilation in the prone position after lung transplantation. Transplant Proc 2012; 44: 2016-2021.

24 Scaravilli V, Grasselli G, Castagna L, et al. Prone positioning improves oxygenation in spontaneously breathing nonintubated patients with hypoxemic acute respiratory failure: a retrospective study. J Crit Care 2015; 30: 1390-1394.

25 Ding L, Wang L, Ma W, et al. Efficacy and safety of early prone positioning combined with HFNC or NIV in moderate to severe ARDS: a multi-center prospective cohort study. Crit Care 2020; 24: 28.

26 Pérez-Nieto OR, Guerrero-Gutiérrez MA, Deloya-Tomas E, et al. Prone positioning combined with high-flow nasal cannula in severe noninfectious ARDS. Crit Care 2020: 24; 114

27 Sun Q, Qiu H, Huang M, et al. Lower mortality of COVID-19 by early recognition and intervention: experience from Jiangsu Province. Ann Intensive Care 2020; 10: 33.

28 Elharrar X, Trigui Y, Dols AM, et al. Use of prone positioning in nonintubated patients with COVID-19 and hypoxemic acute respiratory failure. JAMA 2020; 323: 2336-2338.

29 Despres C, Brunin Y, Berthier F, et al. Prone positioning combined with high-flow nasal or conventional oxygen therapy in severe Covid-19 patients. Crit Care 2020; 24: 256.

$30 \mathrm{Xu}$ Q, Wang T, Qin X, et al. Early awake prone position combined with high-flow nasal oxygen therapy in severe COVID-19: a case series. Crit Care 2020; 24: 250.

$31 \mathrm{Ng}$ Z, Tay WC, Ho CHB. Awake prone positioning for non-intubated oxygen dependent COVID-19 pneumonia patients. Eur Respir J 2020; 56: 2001198.

32 Damarla M, Zaeh S, Niedermeyer S, et al. Prone positioning of nonintubated patients with COVID-19. Am J Respir Crit Care Med 2020; 202: 604-606.

33 Reddy MP, Subramaniam A, Lim ZJ, et al. Prone positioning of non-intubated patients with COVID-19 - a systematic review and meta-analysis. medRxiv 2020; preprint [https://doi.org/10.1101/2020.10.12.20211748].

34 Sartini C, Tresoldi M, Scarpellini P, et al. Respiratory parameters in patients with COVID-19 after using noninvasive ventilation in the prone position outside the intensive care unit. JAMA 2020; 323: 2338-2340.

35 Coppo A, Bellani G, Winterton D, et al. Feasibility and physiological effects of prone positioning in non-intubated patients with acute respiratory failure due to COVID-19 (PRON-COVID): a prospective cohort study. Lancet Respir Med 2020; 8: 765-774.

36 Caputo ND, Strayer RJ, Levitan R. Early self-proning in awake, non-intubated patients in the emergency department: a single ED's experience during the COVID-19 pandemic. Acad Emerg Med 2020; 27: 375-378.

37 Thompson AE, Ranard BL, Wei Y, et al. Prone positioning in awake, nonintubated patients with COVID-19 hypoxemic respiratory failure. JAMA Intern Med 2020; 180: 1537-1539.

38 Hallifax RJ, Porter BM, Elder PJ, et al. Successful awake proning is associated with improved clinical outcomes in patients with COVID-19: single-centre high-dependency unit experience. BMJ Open Respir Res 2020; 7: e000378.

39 Padrão EMH, Valente FS, Besen BAMP, et al. Awake prone positioning in COVID-19 hypoxemic respiratory failure: exploratory findings in a single-center retrospective cohort study. Acad Emerg Med 2020; 27: 1249-1259.

40 Ferrando C, Mellado-Artigas R, Gea A, et al. Awake prone positioning does not reduce the risk of intubation in COVID-19 treated with high-flow nasal oxygen therapy: a multicenter, adjusted cohort study. Crit Care 2020; 24: 597.

41 Jagan N, Morrow LE, Walters RW, et al. The POSITIONED study: prone positioning in nonventilated coronavirus disease 2019 patients - a retrospective analysis. Crit Care Explor 2020; 2: e0229.

42 Prud'homme E, Trigui $\mathrm{Y}$, Elharrar $\mathrm{X}$, et al. Effect of prone positioning on the respiratory support of non-intubated patients with COVID-19 and acute hypoxemic respiratory failure: a retrospective matching cohort study. Chest 2021; in press.

43 Telias I, Katira BH, Brochard L. Is the prone position helpful during spontaneous breathing in patients with COVID-19? JAMA 2020; 323: 2265-2267.

44 Li J, Pavlov I, Laffey JG, et al. Meta-trial of awake prone positioning with nasal high flow therapy: invitation to join a pandemic collaborative research effort. J Crit Care 2020; 60: 140-142. 